\title{
X-RAY AND ELECTRON-OPTICAL CHARACTERIZATION OF ZnTe/CdTe AND ZnTe/GaAs EPITAXIAL LAYERS OBTAINED BY THE MBE METHOD
}

\section{J. Auleytner, Z. Dziuba, J. Górecka, J. Pelka and K. Regiński} Institute of Plysics; Polish Academy of Sciences

Al. Lotników 32/46, 02-668 Warszawa, Poland

\begin{abstract}
$\mathrm{X}$-ray diffraction topography (Bragg diffraction) and X-ray rocking curve measurements were used to study the perfection and structural properties of ZnTe epitaxial layers on the CdTe and GaAs substrates. ZnTe epitaxial layers on CdTe were grown by MBE method by using a machine made in the Institute of Physics of the Polish Academy of Sciences. The ZnTe layers on GaAs were produced on the other, factory-made MBE system. The comparison between the $\mathrm{X}$-ray topograplical images of the substrate and epitaxial layer shows that imperfections on the substrate surface cause imperfections in the epitaxial layer. The results of double-crystal diffractometry measurements show that the perfection of the layer on the GaAs substrate is higher than that on the CdTe. The presence of microtwining in the $\mathrm{ZnTe}$ layer on the CdTe substrate was confirmed by RHEED measurements. The X-ray standing wave fluorescent spectra were also measured for the samples.

PACS numbers: $61.10 .-\mathrm{i}, 61.14 . \mathrm{Hg}, 68.55 .-\mathrm{a}$
\end{abstract}

\section{Introduction}

Production of good quality epitaxial layers based on binary substrates belongs to the main trends of both contemporary solid state physics and semiconductor technology. Therefore, development of diagnostic methods for characterization of the layer is from this point of view of high importance, and can open new possibilities for real structure studies of thin layers. There have been done numerous papers on semiconductor epitaxial layer structure (see e.g. [1] and references therein). Ilowever, our knowledge of these problems is still far incomplete, especially when regarding relatively thick layers.

The aim of this work was to test what information can be reached when applying various disturbance-free techniques based on X-ray and electron diffraction. Here, the X-ray topography, the RIIEED, the rocking curve measurements, and also the Zn fluorescence yield excited by XSW wave field were applied to study the structure of two $\mathrm{ZnTe}$ epitaxial layers on various substrates. 


\section{Sample preparation}

ZnTe (111) epitaxial layers on CdTe (111) were grown by MBE method by a machine constructed in the Institute of Physics of the Polish Academy of Sciences. Two effusion cells were used simultaneously as sources of molecular beams the $\mathrm{ZnTe}$ effusion cell with the temperature $590^{\circ} \mathrm{C}$ and $\mathrm{Zn}$ effusion cell with the temperature $340^{\circ} \mathrm{C}$. Thus the $\mathrm{ZnTe}$ growth process took place under excess of $\mathrm{Zn}$ flux. The growth process was interrupted after 20 minutes. Pressure in the chamber during the process monitored by vacuum gauge was of the order of $10^{-6} \mathrm{~Pa}$. The substrate temperature during the growth process was about $310^{\circ} \mathrm{C} . \mathrm{CdTe}$ substrate wafer was cut from the crystal produced from vapor phase using modified Markow method [2], and chemo-mechanically polished in a solution of bromine in methanol. The thickness of the $\mathrm{ZnTe} / \mathrm{CdTe}$ epitaxial layer determined from the $\mathrm{X}$-ray absorption experiments was $4800 \pm 300 \AA$. The interference microscopy gave the value of about $3000 \AA$. The discrepancies can be explained when regarding differences between the two methods.

ZnTe (111) layer on GaAs (111) was produced on the other, commercial MBE system at the Institute of Crystallography of the IIumboldt University in Berlin. The thickness of the $\mathrm{ZnT} / \mathrm{GaAs}$ epitaxial layer was estimated from the technological parameters to be of about $9500 \AA$ and confirmed by optical measurements.

\section{Experimental}

\subsection{X-ray topography}

X-ray diffraction traverse topography (Bragg diffraction) was used to investigate $\mathrm{ZnTe}$ epitaxial layer on Cd'Te and GaAs substrates. The topographs were obtained using the Lang camera with a conventional X-ray source (see Fig. 1A, B). They were registered on AGFA Gevacrt D5 films. Due to a big difference in lattice constants between CdTe and $\mathrm{Zn}^{\prime} \mathrm{Te}\left(a_{\mathrm{CdTe}}=6.48 \AA, a_{\mathrm{ZnTe}}=6.10 \AA\right)$ and between GaAs and $\mathrm{ZnTe}\left(a_{\mathrm{GaAs}}=5.65 \AA\right)$ it was possible to obtain topographs of the substrate surface and epitaxial layer separately (see Table). The reflection (333)

\section{TABLE}

Bragg angles for $\mathrm{Cu} K_{\alpha_{1}}$ radiation.

\begin{tabular}{c|c|c}
\hline \hline Reflection & Material & $O_{\mathrm{B}}\left[{ }^{\circ}\right]$ \\
\hline \multirow{4}{*}{333} & GaAs & 45.10 \\
\cline { 2 - 3 } & CdTe & 38.14 \\
\cline { 2 - 3 } & ZnTe & 41.00 \\
\hline \multirow{2}{*}{044} & CdTe & 42.25 \\
\cline { 2 - 3 } & ZnTe & 45.58
\end{tabular}




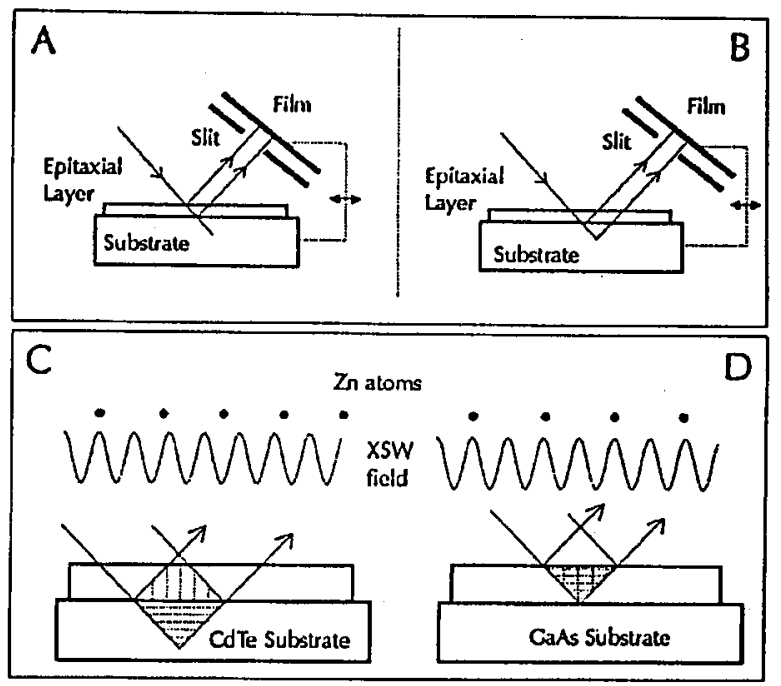

Fig. 1. Sketch of experimental situations in the topographic and XSW measurements. (B), (D) X-ray topographs - geometry of experiment. (A) Topograph of an epitaxial layer; (B) topograph of a substrate. (C), (D) Rocking curve and XSW measurements of $\mathrm{ZnTe} / \mathrm{CdTe}$ and $\mathrm{ZnTe} / \mathrm{GaAs}$, respectively.

$\boldsymbol{A}$

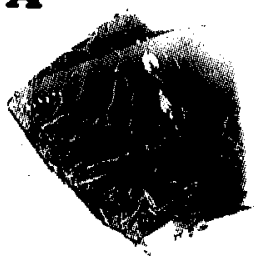

B

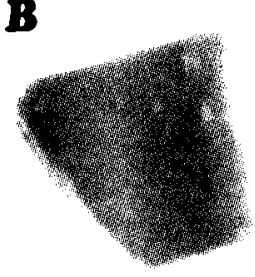

C

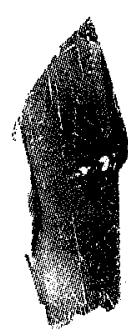

$\mathbf{D}$

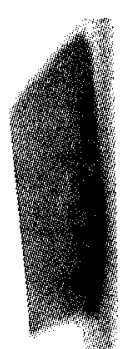

$\mathbf{E}$

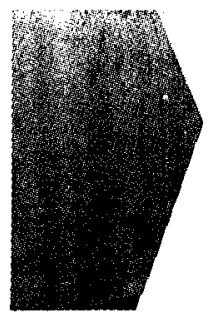

$\boldsymbol{F}$

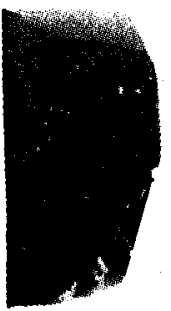

Fig. 2. X-ray topographs - $\mathrm{Cu} K_{\alpha_{1}}$ radiation. (a) $\mathrm{ZnTe} / \mathrm{Cd} \mathrm{Te}$, reflection 333 - substrate; (b) ZnTe/CdTe, reflection 333 - epitaxial layer; (c) ZnTe/CdTe, reflection 440 - substrate; (d) ZnTe/CdTe, reflection 410 - epitaxial layer; (e) ZnTe/GaAs, reflection 333 - substrate; (f) ZnTe/GaAs, reflection 333 - epitaxial layer. 
(symmetrical diffraction) and (440) (asymmetrical diffraction) for $\mathrm{Cu} K_{\alpha_{1}}$ radiation were used in case of the $\mathrm{ZnTe}$ layer on the CdTe substrate and symmetrical reflection (333) in case of $\mathrm{ZnTe}$ layer on the GaAs substrate. The topographs are shown in Fig. 2a-f. In case of the CdTe substrate only part of the surface was covered by the $\mathrm{ZnTe}$ epitaxial layer. The brighter regions visible on the topographs of the CdTe substrate (Fig. 2a, c) were caused by photoclectric absorption of the incident and diffracted radiation by the ZnTe layer (it should be stressed that the ZnTe layer gives no contribution to diffraction for these topographs) and by deformation of the lattice of the substrate caused by presence of the epitaxial layer.

The $\mathrm{ZnTe}$ epitaxial layers have the same orientation as the substrates. The images of the layers (Fig. 2b, d, f) are "diffused" because of strains and of slightly disoriented micrograins present in the layers. Comparison between the images of the substrate and of the epitaxial layer shows that imperfections on the substrate surface are inherited by imperfections in the epitaxial layer.

\subsection{Study by RIIEED}

The reflection high-energy electron diffraction (RIIEED) technique was applied to characterize the crystal structure of the $\mathrm{Zn} T e / \mathrm{Cd}^{\prime} \mathrm{Te}$ epitaxial film. The
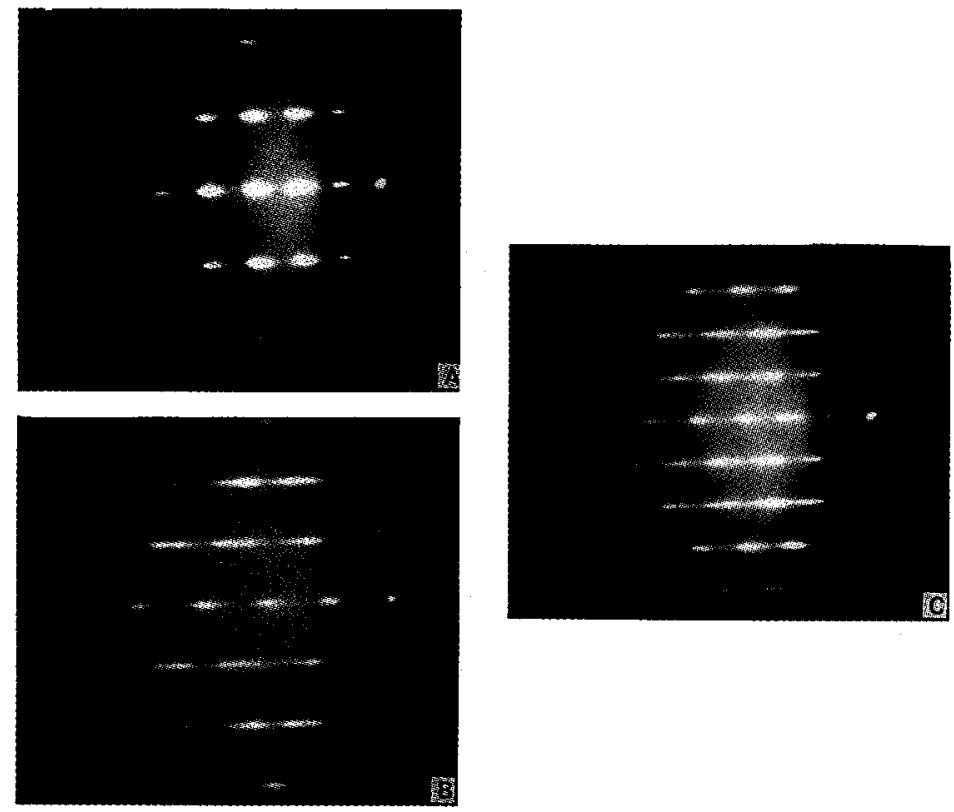

Fig. 3. (A) RIIEED pattern from (111) surface of the ZnTe/CdTe epitaxial layer, [127] azimuth, energy of electron beam $50 \mathrm{kcV}$, incidence angle $\theta=1^{\circ}$; (B) RIIEED pattern from (111) surface of the ZnTe epitaxial film, [110] azimuth, cnergy of electron beam $25 \mathrm{keV}$, incidence angle $\theta=1^{\circ}$; (C) RIIEED pattern from (111) surface of the ZnTe epitaxial film, [110] azimuth, energy of clectron beam $50 \mathrm{keV}$, incidence angle $\theta=1^{\circ}$. 
apparatus used was the EMR-102 diffractometer supplied with an electron gun working at $25 \mathrm{keV}$ and $50 \mathrm{keV}$. The system was equipped with the precise sample manipulator that enabled to change the incidence angle $\theta$ of the electron beam within a very large range and the azimuth angle in the range $(0 \ldots 2 \pi)$. For the grazing angle of incidence $\left(0=1^{\circ}\right)$ and beam energy mentioned above the RIIEED pattern refers to layers situated at deptlis of a few nanometers, thus allowing to observe the structure of the epitaxial layer from the surface to this depth. Figure $3 A$ shows a RIIEED pattern taken in the [121] azimuth. The angle of incidence is $\theta=1^{\circ}$ and the energy of electron beam is $50 \mathrm{keV}$. The diffraction picture reveals a system of intense Bragg reflections and weak diffraction rings near the direct beam. Such a picture is characteristic of a monocrystalline structure with a small admixture of polycrystalline phase. The broadening of the Bragg spots is due to twin formation on $\{111\}$ planes, as it is evident from Figs. 3B and $3 \mathrm{C}$.

The above conclusions are confirmed by diffraction pictures presented in Fig. 3B (beam energy $25 \mathrm{keV}$ ) and Fig. 3C (beam energy $50 \mathrm{keV}$ ). Both the patterns are taken in the [110] azimuth and at the angle of incidence $\theta=1^{\circ}$. The diffraction pictures reveal splitting of the Bragg reflections into pairs of spots, which is characteristic of twin formation on $\{111\}$ planes. Some small admixture of polycrystalline phase is also seen.The geometry of the diffraction pattern does not depend on beam energy (and as a consequence on penetration depth), so we can conclude that the topmost surface layer is homogeneous in the direction perpendicular to its surface.

\subsection{Rocking curve measurements}

The rocking curve and XSW measurements of the samples were performed by precise double-crystal spectrometer with the classical X-ray tube using the Mo $K_{\alpha}$ radiation and $\mathrm{Si}(111)$ monochromator. The rocking curve for the $\mathrm{ZnTe} / \mathrm{CdTe}$ is presented in Fig. $4 \Lambda$, and it arises from the substrate (see Fig. 1C for explanation). It is evidently broader than expected for the ideal crystal (about 5 times). At its higher-angle side there exists also an additional structure, placed about

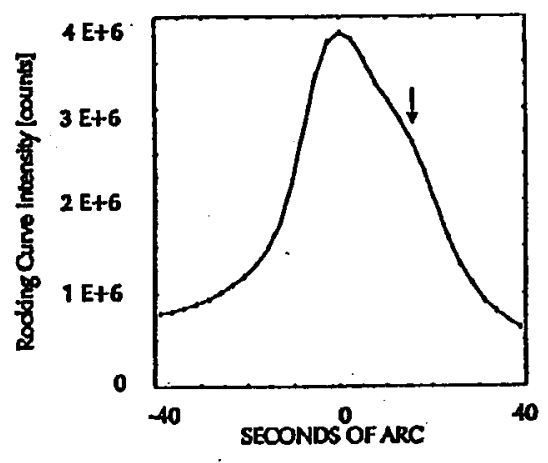

Fig. 4. Rocking curve of the $\mathrm{ZnTe} / \mathrm{CdTe}$ sample. 


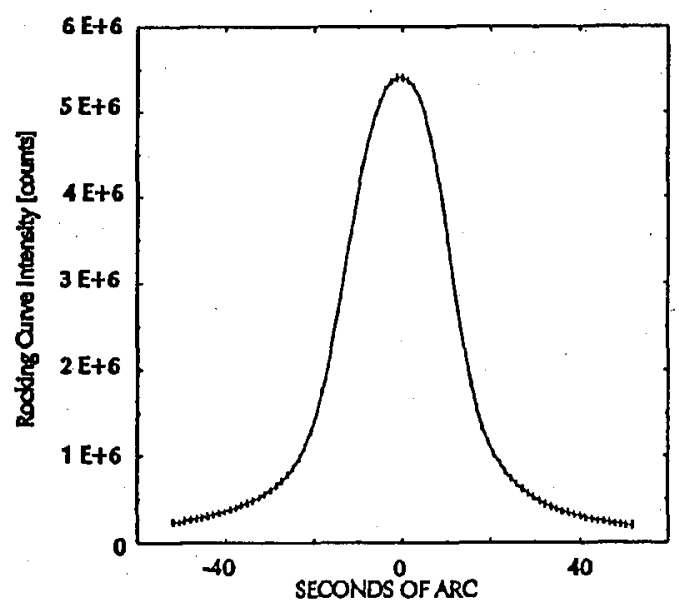

Fig. 5. Rocking curve of the ZnTe/GaAs sample.

15 seconds of arc from the main peak. Assuming that the experimental rocking curve is composed of two pealss similar in shape, we can estimate the intensity of the second peak as less than 20 percent of the main peak. The general shape and width of the curve reflects a large density of defects and strains in the lattice, probably due to interface creation with significant lattice mismatch between $\mathrm{ZnTe}$ and CdTe. The rocking curve from the epitaxial layer was very diffused and weak for this sample, and it is not reported here. The rocking curve for the $\mathrm{ZnTe} / \mathrm{GaAs}$ comes from the epitaxial layer itself (see Fig. 1D). It is slown in Fig. 5 (solid line). Although its width is also higher than in ideal crystal it is narrower comparing to that one for the previous sample. Its sliape is "smooth" without any pronounced secondary structure. It can suggest a higher level ordering in the layer. The fluorescence yield excited with the XSW wave field was also recorded at the same time with the rocking curves by the Si:Li detector. The problems of interpretation of the XSW spectra will be discussed later.

\section{Discussion of results and conclusions}

The comparison between the X-ray topogr'phical images of the substrate and epitaxial layer shows that imperfections of the epitaxial layer are inherited from imperfections on the substrate surface. The results of double-crystal diffractometry measurements show that the perfection of the layer on GaAs substrate is higher than that on CdTe. The presence of microtwining in the ZnTe/CdTe sample was confirmed by RIIEED measurements. The ZnTe/Cd'Te rocking curve is evidently broadened with respect to the llieoretical one. A pronounced structure on its higher-angle side, of about $15^{\prime \prime}$ of arc from the main maximum is observed. It can suggest that the signals from at least two regions differing with lattice constant and/or ordering are superimposed. It is probable that the smaller component of the rocking curve arises from the intermediate layer created near the substrate surface. 
The fluorescence yield excited with the XSW wave field was also recorded at the same time with the rocking curves by the Si:Li detector. However, the interpretation of the spectra for the samples investigated in this work is practically impossible, and the spectra themselves are not reported here. There are important differences in creation of the XSW signal between the two samples in our measurements. It is explained schematically in Fig. 1C, D. For the ZnTe/CdTe sample the $\mathrm{XSW}$ wave field is created entirely in the substrate layer having, as a consequence, periodicity of this layer. This wave field excites then the $\mathrm{Zn}$ atoms, which are positioned in the upper layer. Because of about $4 \%$ mismatch in lattice parameters between the substrate and the $\mathrm{Zn}$ Te layer, the XSW wave field is incommensurate with the nodal positions of the epitaxial layers. In case of the $\mathrm{ZnTe} / \mathrm{GaAs}$ sample measurements both the XSW wave field and the fluorescence from $\mathrm{Zn}$ atoms are created in the same epitaxial layer. No mismatch between the XSW wavelength and lattice constant is expected for this sample, which could simplify the problem of interpretation. It should be stressed that both layers containing the $\mathrm{Zn}$ atoms were thick enough to a verage the XSW fluorescent signal.

It has been proven that in such complicated physical situation an attempt of numerical modelling based on a simple XSW algorithm described elsewhere $[3,4]$ fails. Although in both cases the fitting algorithm gives some concrete results for the $\mathrm{Zn}$ atomic positions and amounts of coherent fractions, they disagree with expectations based on structural models for the crystals, which assume that significant amount of $\mathrm{Zn}$ atoms should occupy their nodal positions. In addition, the fitting procedure was not able to distinguish among various, even very different positions and coherent fractions.

It has been shown that complementary studies with the use of X-ray and electron diffraction methods performed in this work for the ZnTe MBE epitaxial layers on two various substrates enable us the more complete characterization of their structure. The comparison between the X-ray topographical images of the substrate and epitaxial layer shows that imperfections on the substrate surface cause imperfections in the epitaxial layer. The results of double-crystal diffractometry measurements show that the perfection of the layer on GaAs substrate is higher than that on Cd'Te. The presence of microtwining in ZnTe layer on CdTe substrate has been confirmed by RIIEED measurements. The interpretation of the XSW fluorescent spectra, mcasured for the samples, was found to be very difficult. Two of the most important reasons were the complexity of physical situation in the samples and insufficient statistics of the measurements due to small brightness of the conventional X-ray source. It seems that applying of the synchrotron radiation for such studies would provide spectra of much better quality, and it would give a possibility to precise from the models of atomic positions distribution and their coherent fractions.

\section{Acknowledgnonts}

The authors wish to thank Dr. A. Nadolny and Dr. J. Sadowski for preparing the $\mathrm{ZnTe} / \mathrm{Cd}$ Te sample and their help in its characterization. 
This work was partially sponsored by the State Committee for Scientific Research (Republic of Poland) (grant no. 337139203 and PB 935/2/91).

\section{References}

[1] C. Giannini, L. Tapfer, S. Lagomarsino, J.C. Boulliard, A. Taccoen, B. Capelle, M. Ilg, O. Brandt, K.H. Ploog, Phys. Rev. B 48, 496 (1993).

[2] K. Grasza, U. Zuzga-Grasza, A. Jędrzejczak, R.R. Gałązka, J. Majewski, A. Szadowski, E. Grodzicka, J. Cryst. Growlh 123, 519 (1992).

[3] J. Pełka, J. Auleytner, Acla Phys. Pol. A 82, 163 (1992).

[4] M.V. Kovalchuk, V.G. Kohn, Usp. Fiz. Nauk 149, 69 (1986). 